\title{
A MODEL OF MOLECULAR EMISSION IN BIPOLAR FLOWS
}

\author{
Sylvie Cabrit and Claude Bertout \\ Institut d'Astrophysique de Paris \\ 98bis Bd Arago \\ 75014 PARIS \\ FRANCE
}

\begin{abstract}
We present a model of $\mathrm{CO} \mathrm{J}=1 \rightarrow 0$ line formation in accelerated bipolar outflows. We find that the integrated intensity maps can be divided into four categories, depending on the view angle and the flow opening angle. We then show that no accurate values of the flow parameters can be derived from the $\mathrm{CO}$ observations without careful modelling of the outflow properties.
\end{abstract}

\section{INTRODUCTION}

Recent millimeter and optical studies of star-forming regions have provided strong evidence that many young stellar objects are undergoing a phase of energetic, collimated outflow activity (cf. Lada 1985). CO line observations are especially important since they offer a powerful probe of the physical properties, large scale structure, and kinematics of the high-velocity molecular gas.

In order to exploit the wealth of information contained in $\mathrm{CO}$ observations, we have developed a code which computes the excitation and the rotational line profiles of $\mathrm{CO}$ in axial symetry in the presence of supersonic accelerated velocity fields. Our first investigation was emission from a steady-state radial outflow expanding in two biconical lobes. This short contribution reviews our main assumptions and implications for high-velocity $\mathrm{CO}$ excitation and outflow geometry. A complete analysis of our results can be found in Cabrit and Bertout (1986).

\section{MAIN ASSUMPTIONS}

\subsection{Flow geometry}

Although many molecular flows have an obvious bipolar geometry, a major uncertainty is whether the observed lobes are filled with high-velocity gas or trace the surface of an expanding hollow shell. While the first case would yield wide, single-peaked profiles similar to the observed ones, the second should produce narrower, doublepeaked profiles when the line of sight crosses two distinct thin layers, unless the turbulent velocity within the shell is of the order of magnitude of the outflow velocity, or the shell is made of unresolved clumps moving at different velocities. In at least one flow, L1551-IRS5 (Moriarty-Schieven et al.1986), a bright- rim structure in the high- 
velocity gas is seen which is suggestive of the shell geometry. At this time, we present the results for the filled cone model. We assume that the high-velocity gas fills a bicone of opening angle $2 \theta_{\max }$, inner radius $r_{\min }$ and outer radius $r_{\max }$, and that the velocity and density laws are exponential in $(1 / r)$ with exponents $\alpha$ and $\delta$. Mass conservation within the flow required that $\delta=2-\alpha$. Two cases were investigated: a constant velocity outflow with $\alpha=0$ and a linearly accelerated outflow with $\alpha=-1$.

\subsection{CO excitation}

In conditions typical of molecular clouds, the $\mathrm{CO}$ rotational lines are excited mainly by radiative transitions and collisions with hydrogen molecules. The sources of radiation external to the high-velocity gas are the thermal emission of dust grains, the line emission of the static ambient cloud, the photospheric emission from the central star, and the cosmic background emission at $2.7 \mathrm{~K}$. Computations of the ratio of dust grains to molecular gas emissivity show that the effect of dust-to-gas radiative coupling on the transfer of the first two rotational lines of $\mathrm{CO}$ is always negligible, but that it may be important for higher transitions. The static cloud line radiation, being Doppler-shifted in the high-velocity gas rest frame, cannot contribute to the $\mathrm{CO}$ excitation in the flow and did not appear in our computations. For moderate high-velocity CO optical depths, we found that heating by stellar radiation was noticeable only at distances $r<r_{*}\left(T_{*} / 10,000 \mathrm{~K}\right)^{1 / 2}$. Since the $\mathrm{CO}$ observations suggest that high-velocity emission originates at distances greater than $10^{-2} \mathrm{pc}$ (Goldsmith et al. 1984, MoriartySchieven et al. 1986), we also neglected the stellar contribution and considered that the high-velocity gas was heated only by collisions and absorption of local or cosmic background photons.

Because the outflow velocities inferred from molecular observations $(\mathrm{v} \geq 10 \mathrm{~km}$ $\left.\mathrm{s}^{-1}\right)$ are much larger than the CO thermal velocity $\left(v_{\text {th }}=0.02 \sqrt{T_{k}} \mathrm{~km} \mathrm{~s}^{-1}\right)$, we adopted the fast-flow approximation to compute NLTE level populations, but did not use it for the emergent profiles, which were integrated exactly. This hybrid method has been shown to give excellent results for fast flows (Bastian et al. 1980). The CO excitation conditions at a given point in the flow are then uniquely determined by the values of the following independent parameters: $\mathrm{T}_{\mathrm{k}}, \mathrm{n}_{\mathrm{H}_{2}}, \alpha$, and the local optical depth $\mathrm{k}(\mathrm{r})=8 \pi^{3} / 3 \mathrm{~h} \mu^{2} \mathrm{n}_{\mathrm{CO}} \mathrm{r} / \mathrm{v}(\mathrm{r})$.

\section{PHYSICAL IMPLICATIONS}

\section{1. $\mathrm{CO}$ excitation}

In agreement with theoretical expectations, we found that the $\mathrm{J}=1 \rightarrow 0$ transition was thermalized only for:

$$
\left(\mathrm{n}_{\mathrm{H}_{2}}\right)^{2}>530 \mathrm{v}\left(\mathrm{km} \mathrm{s}^{-1}\right) /\left(\mathrm{A}_{\mathrm{CO}} \mathrm{r}(\mathrm{pc})\right) \mathrm{cm}^{-6} \text {. }
$$

In a typical molecular flow, where $A_{\mathrm{CO}} \sim 10^{-4}, \mathrm{r} \sim 0.1 \mathrm{pc}, \mathrm{v} \sim 10 \mathrm{~km} \mathrm{~s}^{-1}$, this condition becomes :

$$
\mathrm{n}_{\mathrm{H}_{2}}>2.510^{4} \mathrm{~cm}^{-3} \text {. }
$$

The absence of detectable high-velocity CS emission in the observed CO lobes seems to indicate that in molecular flows the mean density lies below this critical value. NLTE calculations of the CO excitation are thus required if most of the high-velocity emission is formed in an extended region of moderate density. 


\subsection{Flow geometry}

For a radial biconical outflow, four types of configurations can be distinguished, depending on the values of $\theta_{\max }$ and $i$, the angle between the flow axis and the line of sight.

Case 1: $\mathbf{i}<\theta_{\max }$ and $\mathbf{i}<\pi / 2-\theta_{\max }$, is illustrated in Fig.1a ( $\mathrm{i}=10^{\circ}, \theta_{\max }=$ $30^{\circ}$ ). The two cones are seen nearly face-on, so that the contours of blue- and redshifted integrated intensity are circular and overlapping.

Case 2: $\mathbf{i}>\theta_{\max }$ and $\mathbf{i}<\pi / 2-\theta_{\max }$, occurs only if $\theta_{\max }<45^{\circ}$ and is illustrated in Fig. $1 \mathrm{~b}\left(\mathrm{i}=50^{\circ}, \theta_{\max }=30^{\circ}\right)$. The blue and red-shifted lobes are now spatially separated and the bipolar structure of the flow is apparent.

Case 3: $\mathrm{i}>\theta_{\max }$ and $\mathrm{i} \geq \pi / 2-\theta_{\max }$, is presented in Fig. $1 \mathrm{c}\left(\mathrm{i}=80^{\circ}, \theta_{\max }=\right.$ $30^{\circ}$ ). The two cones again appear as two distinct lobes but each one is now partly blue-, partly red-shifted.

Case 4: $\mathbf{i}<\theta_{\max }$ and $\mathbf{i} \geq \pi / 2-\theta_{\max }$, occurs only for $\theta_{\max }>45^{\circ}$ and is illustrated in Fig. $1 \mathrm{~d}\left(\mathrm{i}=50^{\circ}, \theta_{\max }=60^{\circ}\right)$. It is simply a Case 3 where the two lobes are still overlapping due to their larger opening angle.

1a)

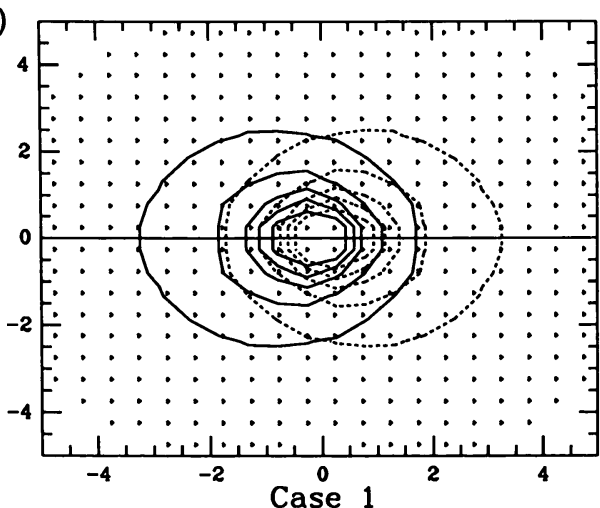

1c)

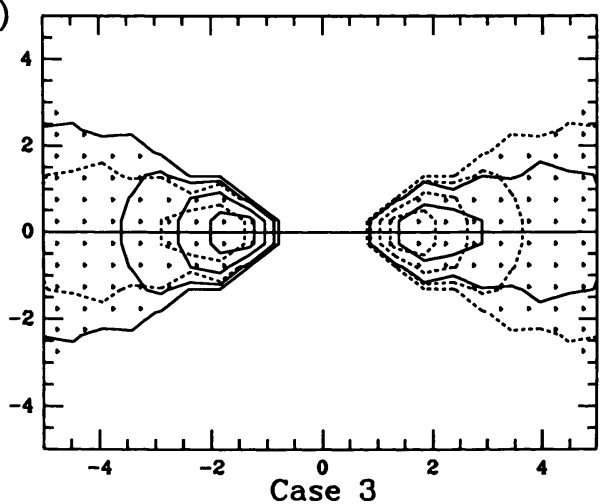

1b)

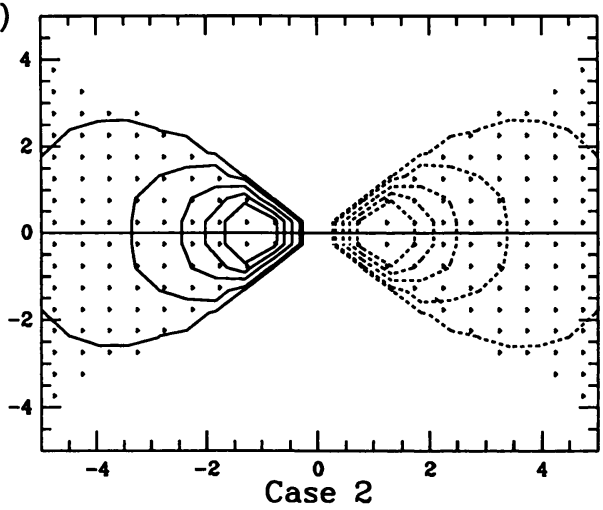

1d)

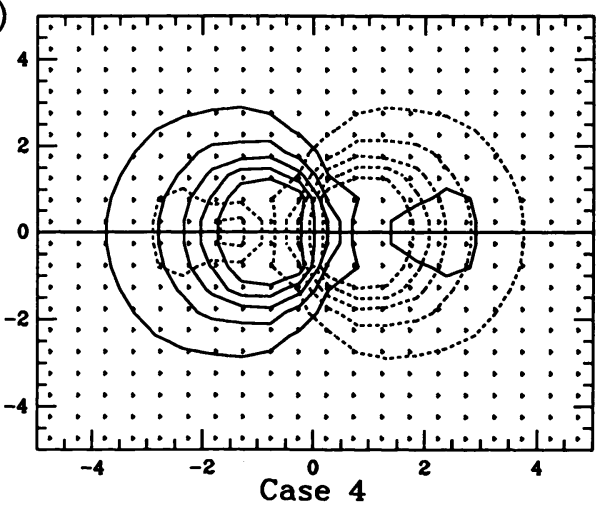

Figure 1a to 1d (left to right and top to bottom). Synthetic contour maps of blueshifted (solid lines) and red-shifted (dashed lines) CO J=1 $\rightarrow 0$ integrated intensity computed for $\mathrm{n}_{\mathrm{H}_{2}}\left(\mathrm{r}_{\min }\right)=10^{4} \mathrm{~cm}^{-3}, \mathrm{~T}_{\mathrm{k}}=10 \mathrm{~K}, \mathrm{k}\left(\mathrm{r}_{\min }\right)=100, \alpha=-1, \mathrm{r}_{\max }=10 \times \mathrm{r}_{\min }$, illustrating the four flow configurations defined above (see text for details). 
Although Cases 1 and 2 reproduce quite well the main aspects of the observed flows (see Lada 1985), Cases 3 and 4 have never been reported, even though they are expected whenever $\theta_{\max }>30^{\circ}$. There are three possible explanations for this lack of detection: First, emission at low radial velocity is undiscernable from the ambient cloud emission, so that Case 3 (resp. Case 4) flows might be classified as Case 2 (resp. Case 1) flows after subtraction of the central low-velocity emission. Second, observations that are attributed to a pair of outflows seen face-on could in fact represent a single Case 3 flow. Finally, most bipolar flows might have $\theta_{\max }<30^{\circ}$. These conclusions remain valid for a radial outflow confined to a conical shell.

Our calculations have shown that the $\mathrm{CO}$ emission is also strongly dependent on the velocity field (Cabrit and Bertout 1986), which makes our model a powerful tool for the study of molecular flows properties.

\section{OBSERVATIONAL UNCERTAINTIES IN THE FLOW PARAMETERS}

In order to test the method commonly used by observers for deriving flow properties, we applied it to our synthetic maps and compared the resulting estimates with the actual parameters of our model.

We found that the assumption of small optical depth underestimates the CO mass and momentum by a factor of 25 at worst, except in flows with low optical depth and high density gradients where they can be overestimated by up to a factor of 2 . The dynamical time scale, obtained by dividing the apparent size of the flow by the maximum observed velocity, is always too small because the flow length is underestimated, especially at small view angles. And if $\alpha=-1$, it can be in error by up to a factor of 20 , because in accelerated flows the velocity at the base of the flow is overestimated. In that case, the rate at which momentum is transferred to the CO flow is overestimated by similar factors, but it can also be underestimated by up to a factor 10 when $\alpha=0$ and the optical depth is large.

The values of the rates of momentum and energy input in the flow derived from the $\mathrm{CO}$ observations must therefore be considered with extreme caution.

\section{CONCLUSION}

This model gives a better understanding of what can be expected and deduced from the geometry and emission characteristics of molecular outflows. Our code is now being improved to handle more complex geometries (thin shells) and velocity fields (decelerated flows), which will be investigated in our next papers. We will also attempt detailed comparisons of our computations with recent high-resolution $\mathrm{CO}$ observations. Only after such a study will we understand the kinematics of bipolar flows and the constraints they put upon mass-loss mechanisms in pre-main sequence stars.

\section{REFERENCES}

Bastian, U., Bertout, C., Stenholm, L., Wehrse, R. 1980, Astr. Ap. 86, 105

Cabrit, S., Bertout, C. 1986, Ap. J. 307, 313

Goldsmith, P.F., Snell, R.L., Hemeon-Heyer, M., Langer, W.D. 1984, Ap. J. 286, 599

Lada, C.J. 1985, Ann. Rev. Astr. Ap. 23, 267

Moriarty-Schieven, G., Snell, R.L., Strom, S.E., Schloerb, F.P., Strom, K.M. 1986, Submitted to Ap. J. 